\title{
Reactive devices. Maker experience as a form of pedagogical innovation in formation of industrial designers
}

XXIV International Conference

of the Iberoamerican Society

of Digital Graphics

Medellin | Colombia

\author{
Sebastián Martini \\ Universidad Nacional del Litoral | Argentina | sebamartinidis@gmail.com \\ Mauro Chiarella \\ CONICET-Universidad Nacional del Litoral | Argentina | mchiarella@hotmail.com
}

\begin{abstract}
In this presentation, the reflections about the application of an innovative cathedra experience for the formation of industrial designers will be exposed. It revolved around the development of what the cathedra team defines as "reactive devices", highlighting the role of the design into an educational proposal that is based on the principles of Maker culture. In this experience converge knowledge from different areas of knowledge, through a synergy of conventional and disruptive technologies that allow novel approaches of capture, analyze and understanding of the reality in the searching of a design for the transformation.
\end{abstract}

Keywords: Maker culture; PBL; Curriculum, Interdisciplinary; Digital fabrication

\section{INTRODUCCIÓN}

Se presenta aquí un trabajo multidimensional en torno a los aspectos metodológicos y epistémicos de la cultura académica del diseño industrial (CADI). El estudio propone una reflexión sobre los aportes que se pueden derivar de las acciones desarrolladas dentro de la cultura Maker en el diseño de prácticas de enseñanza para la formación de diseñadores industriales. Esta estrategia que se plantea fue necesaria para construir, desde una mirada divergente, una composición innovadora sobre los modos de generar y transmitir conocimientos dentro de esta disciplina proyectual. De esta forma, del mismo modo en que los diseñadores emprendemos el proceso de diseño creativo, el cual se despliega durante un intervalo de tiempo, este proceso también requirió de distintos momentos para su definición. Cómo dice Forster (2019): "en el espacio temporal del proceso creativo es donde se producen las operaciones y las relaciones, es en ese transcurrir donde se atraviesan y manipulan los estados de potencia, donde se construye y surge la creación de lo nuevo".

De esta manera hemos iniciado, durante los últimos años, trabajos de búsqueda y experimentación que hoy forman parte de este estudio y que previamente han sido socializados en distintos eventos académicos. Estos espacios, en particular el congreso internacional de SiGraDi han permitido instancias previas de investigación y producción que hicieron posible el desarrollo conceptual que aquí describiremos. Dichos estudios se fueron orientando hacia la exploración del curriculum y las prácticas de enseñanza de la carrera de Diseño Industrial (DI), donde desde sus comienzos nos venimos desempeñando como docentes en diferentes cátedras de la misma. Particularmente comenzamos a indagar sobre las características de los aprendizajes que se derivaban de prácticas de enseñanza Maker, bajo una mirada que integra el pensamiento curricular para la formación de diseñadores industriales.

Ahora bien, del cruce entre las relaciones de las experiencias provenientes de la cultura Maker y las correspondientes al DI propiamente dichas, situamos el siguiente razonamiento: $\mathrm{Si}$ las tecnologías digitales irrumpen con una fuerte inercia en la sociedad global, volviéndose ante nuestros ojos como la forma preeminente de generar, distribuir y transmitir conocimiento, ¿qué sucede con las propuestas educativas en torno a la enseñanza universitaria del diseño industrial? ¿Cómo se organizan y definen los contenidos, perspectivas y metodologías en los planes de estudio actuales? ¿Podría pensarse que por fuera de las estructuras académicas formales se encuentran caminos para una mayor reflexividad y creación de nuevas prácticas de enseñanza? Es en este marco que planteamos lo Maker dentro de la dinámica curricular de la carrera en diseño industrial. Por esto nos preguntamos: ¿Cuáles son las características epistemológicas y metodológicas de la cultura Maker? ¿Qué posibilidades existen para generar otras estructuras y prácticas de enseñanza que incorporen esas características en la carrera de diseño industrial? Como docentes de la carrera nos interesa pensar espacios donde se puedan conjugar las innovaciones científicotecnológicas con el desarrollo de proyectos educativos basándonos en la cultura Maker como una posibilidad innovadora de captura, análisis y comprensión de la realidad. De esta forma aspiramos a formar sujetos con los conocimientos necesarios para los desafíos venideros que hoy desconocemos. Que puedan adoptar el diseño como un medio transformador para tornar los problemas en oportunidades. El hacer se constituye como elemento 
motivador, el sujeto elije sus propios retos y se responsabiliza de su aprendizaje para resolver los problemas que se presenten.

\section{APROXIMACIONES CONCEPTUALES PARA PENSAR LA FORMACIÓN DE DISEÑADORES INDUSTRIALES}

Cuando hablamos de formación debemos considerar el campo educativo como fenómeno social humano que involucra la transmisión de cierto marco cultural a las nuevas generaciones (Canciano, 2013). Como hemos anticipado, éste artículo describirá una práctica educativa realizada dentro del nivel superior. Para ello, consideramos necesario, asumiéndonos como sujetos de determinación curricular (de Alba, 1991), describir una serie de conceptos básicos que sirvan como marco de referencia para pensar el diseño de nuevos proyectos educativos. Estos además, darán cuenta del complejo entretejido de dimensiones que afectan, ciertas partes, de los sistemas de educación superior (Burton, 1993). Bajo estos criterios describiremos la noción de currículum, como ámbito de conocimiento vinculado a los procesos institucionales de educación; diferenciándolo de plan de estudios, como organización racional de la enseñanza. Finalmente hablaremos de la relación entre currículum y didáctica, entendiéndola como practica que aborda el problema de la enseñanza en la relación docente-estudiantes.

Respecto a la primera noción, cuando se hace referencia a la planificación de contendidos y al ordenamiento de los mismos, comúnmente se alude al término de currículum o plan de estudios. Estas expresiones suponen, por un lado, una visión sobre el recorrido formativo pensado con intencionalidad educativa y, por otro, un modo restringido de considerar al currículum cada vez que se lo asocia únicamente a la idea de plan. Ahora bien, este reconocimiento de determinadas actividades, prácticas $u$ organizaciones sobre los modos en que se estructuran los conocimientos para ser transmitidos conllevan operaciones de reflexión (a veces tácitos) sobre la misma noción de currículum, haciendo emerger, una vez más, la pregunta: ¿Qué se nombra bajo el término "currículum"?

Para comenzar diferenciaremos las nociones de plan de estudios y curriculum, en pos de conocer con mayor profundidad algunas dimensiones del trabajo académico. Furlan (1992) en su conferencia sobre "curriculum y condiciones institucionales", describe que en sus orígenes, ambos términos aludían a la misma idea: "organización racional de los estudios". Así fue hasta el siglo XIX, momento en que el desarrollo industrial acelerado exigió un trabajo educativo intenso para resolver la formación de la emergente sociedad industrial. Los intelectuales, ligados a la práctica educativa, desarrollaron amplios debates en torno a cómo refuncionalizar la escuela que se veía anacrónica frente a las demandas que la economía, la sociedad y la política le exigían al sistema de educación. Frente a ese contexto, los intelectuales comenzaron a considerar que el currículum podría concebirse como un instrumento para adaptar las instituciones a las nuevas demandas. Esto dio inicio a una puja entre las teorías desarrolladas por John Dewey, quien defendía el aprendizaje a partir de la acción, y Franklin Bobbitt quien promovía el modelo fabril derivado del Taylorismo. A pesar de las diferencias entre los autores, ambos cuestionaban la estructura tradicional de la organización de la enseñanza, que era por disciplinas. Cada autor, desde sus perspectivas, proponía nuevas formas que anteriormente no eran tenidas en cuenta. A raíz del debate Dewey-Bobbit surge la problemática de fundamentar los proyectos educativos y de construirlos de modo tal que garanticen la transformación deseada. Esto fue lo que desencadenó que la noción de currículum se abriera y expandiera respecto a la noción de plan. De esta forma, la expresión de currículum alcanza una categoría compleja y polisémica, que puede dar cuenta tanto del recorrido que se va a realizar como así también del recorrido realizado. Como proyecto formativo da sentido a las instituciones educativas en las que encuentra concreción a través de una serie de mediaciones y particularidades. Entonces, según Furlan, la diferencia entre plan de estudios y currículum es que, el primer término hace alusión directa a la planificación de los estudios, volviéndose complejo utilizarlo de otra manera. En cambio, el curriculum, se conforma como una expresión que da cuenta de un recorrido a realizar o ya hecho, una concepción más amplia que la noción de plan, facilitando su adopción para describir procesos más complejos.

Entonces, a partir de la reconstrucción socio-histórica sintéticamente expuesta, podemos afirmar que de las teorías curriculares desarrolladas durante la modernidad nace una perspectiva ampliada que vemos reflejada en distintos autores contemporáneos. Entre ellos, Alicia de Alba (1991), concibe al currículo como una "síntesis de elementos culturales (conocimientos, valores, costumbres, creencias, hábitos), que conforman una propuesta políticoeducativa...", este proyecto se diseña a partir de ser pensado e impulsado "...por diversos grupos y sectores sociales, cuyos intereses son diversos y contradictorios..." (p38). Además propone estudiar el currículo a partir de identificar "aspectos estructurales-formales y procesalesprácticos", como así también considera las "dimensiones generales y particulares que interactúan en el devenir del currículum en las instituciones sociales educativas".

Actualmente, las cuestiones curriculares se han vuelto complejas, requiriendo considerar, de manera integrada, ideas, dimensiones, aspectos, factores y fuerzas diferentes que, en la teoría y la práctica, se combina al tomar decisiones sobre el currículo como proyecto educativo. Gracias al aporte de autores como de Souza Silva (1999); De Alba (2007); Hidalgo (2013); Diaz Barriga (2007), entre otros; conocemos que los modos de organización de los currículos universitarios se ven afectados por una coyuntura global que se caracteriza por grandes cambios políticos, económicos, sociales y culturares. Las nuevas economías se sostienen sobre el progreso científicotecnológico, con el agregado de una sociedad global hiperconectada que exige una investigación dirigida a resolver los problemas complejos que las afectan (pandemias, deterioro ambiental, pobreza, desempleo, violencia, catástrofes)

La complejización del campo curricular en el siglo XXI es acompañada por un incremento en la producción académica que busca siempre resolver los nudos que las teorías van generando. Una de ellas, de interés para nuestro artículo, es la dimensión referida a las formas de estructuración de las propuestas educativas. Alicia W. Camillioni (2016), elabora un texto que busca explicitar las tendencias y formatos en el curriculum universitario. De 
éste quisiéramos destacar las reflexiones que se dan sobre el recorte de contenidos para dar en el grado, cuáles en los posgrados y la noción de actualización o reentrenamiento de profesionales. Respecto a los formatos curriculares, Camillioni describe como nueva tendencia al currículum por competencias y al curriculum basado en problemas. Este último es de especial interés para nosotros, ya que es el modo en que nuestras disciplinas proyectuales basan sus modos de concebir y transmitir los conocimientos. Como veremos más adelante, es bajo esta concepción que basamos el desarrollo de la ejercitación que describiremos. Sintéticamente podemos decir que un currículo basado en problemas "es, específicamente, el diseño que toma el planteo de problemas como estructura principal y eje en la construcción del currículo. Los problemas elegidos son multidisciplinarios y se sustituye la clásica enseñanza por asignaturas separadas, por un currículo estructurado en problemas complejos que se van planteando de manera seriada en el transcurso de los años de formación de los estudiantes" (Camillioni, 2016).

Ahora bien, habiendo realizado una descripción sociohistórica $\mathrm{y}$, habiendo expuesto, sintéticamente, cierto estado del arte sobre el campo del currículum, continuaremos con la descripción de la última de las nociones que mencionamos al comienzo: la relación entre currículum y didáctica. Si entendemos al currículum como una síntesis de elementos culturales, que determinados sujetos sociales consideran relevantes y legítimos transmitir en el ámbito educativo, el docente se convierte en el representante social y el responsable institucional de intervenir en el espacio áulico, valiéndose de múltiples estrategias pedagógicas y didácticas para alcanzar dichas finalidades (Coscarelli and Picco, 2009). La didáctica es concebida como disciplina dedicada a la explicación, comprensión y orientación de la enseñanza, significada como práctica social que se desarrolla en contextos sociohistóricos concretos y no en ámbitos prefigurados. La didáctica se nutre de conocimientos de diversas disciplinas como: la sociología, la historia, la antropología, la psicología y el psicoanálisis, pero busca efectuar sus desarrollos a partir de la exigencia de reflexionar e intervenir en el aula (Edelstein, 2007). Resumidamente podemos decir que de lo curricular se destaca la búsqueda de conocimientos, enfoques pedagógicos y argumentos que lo justifique y, lo didáctico, apunta a redefinir una situación global en la que esos conocimientos son transmitidos. Las relaciones entre la didáctica y el currículum son complejas. Díaz Barriga (2007) advierte que "las propuestas curriculares tienen un desarrollo conceptual que de alguna forma cancela el punto de vista didáctico". Enuncia como problemática la falta de conocimientos sobre las habilidades técnico-profesionales específicas de la profesión docente, perdiéndose de vista la dimensión intelectual de su trabajo, y por ello conduciendo muchas veces a la idea "dosificador" de un plan de estudios.

Para concluir este apartado, podemos sintetizar que las diferentes teorías curriculares se debaten en un intento de articular posiciones con respecto a los requerimientos de la cultura, la sociedad y los conocimientos valiosos y necesarios. Todo ello respecto a los procesos de enseñanza y aprendizajes adecuados a fin de alcanzar las intenciones formativas perseguidas. Si reconocemos que los sujetos se van formando, más allá de la relación individual con un docente, sino a través de múltiples influencias que recibe durante su trayectoria, entonces, el mero hecho de pensar una nueva práctica educativa, asumiéndonos como sujetos de determinación curricular, debería responsabilizarnos sobre la configuración de la propuesta en función del recorrido total del estudiante. Como dice Furlan (1992:8): "Trabajar con la teoría curricular implica estar aceptando decir: aquí es mucho más importante lo que hacemos en común".

A partir de lo desarrollado y, considerando que los currículos sintetizan elementos culturales (conocimientos, habilidades, valores, etc.) que debemos considerar como operadores curriculares, describiremos, a continuación, algunas de las características que hacen a la cultura de la disciplina.

\section{CULTURA ACADÉMICA DE DISEÑO INDUSTRIAL (CADI).}

El diseño industrial, al igual que la cultura Maker, se nutre de distintas áreas de conocimiento. Esto se debe a que la complejidad de variables que los conforman requiere de múltiples saberes y procedimientos que provienen de las artes, la ciencia, la tecnología y las ciencias sociales. En diseño industrial, la modalización de estas áreas de conocimiento conforma su núcleo disciplinar, el cual parte de un sostenido diálogo interdisciplinar, siendo los aspectos proyectuales de diseño su especificidad. Podemos afirmar que la estructura epistemológica del diseño industrial, tiene origen en múltiples áreas del conocimiento; seguramente por ello y por ser una disciplina relativamente joven, no proyecta una identidad definida a la sociedad. Sin embargo, como dice Donoso (2016), "este aparente caos, es el insumo principal para el pensamiento creativo que lo caracteriza".

Los orígenes académicos del diseño industrial como disciplina se remontan a la escuela de Bauhaus creada en 1919, dentro de la cual el estudiante debía experimentar con distintos materiales y tecnologías antes de poder especializarse. De esta forma observamos que desde sus orígenes, y al igual que sucede en el movimiento Maker, al verbo "hacer" se lo considera de forma ampliada y no escindido del sentir y del pensar. Bajo esta forma de saber, los estudiantes acceden a un conocimiento progresivo y medular del diseño, el llamado pensamiento proyectual, entendiendo su práctica y acercándose dialécticamente al pensar y al hacer dentro de esta disciplina. Bajo estos preconceptos se adopta, para este estudio, un verbo "hacer" aumentado, que excede los límites de lo físico, lo manual, considerándolo desde una perspectiva transformadora que nos actualiza durante los procesos de creación. El conocimiento en diseño se encuadra en el hacer poiético, como dice Tonelli (2009), "se ocupa de la producción de entes que todavía no son. Constituyendo conocimientos que tienen la cualidad de pasar de la potencia al acto, que se crean en el mismo momento".

Por su parte, como hemos visto, la tecnología ha tenido y tiene un rol protagónico dentro de la cultura Maker, como así también en diseño industrial. No solo para el desarrollo de la tecnología en sí misma, sino también como constrictora de las posibilidades formales que pueden fabricarse, y por ende, pensarse. Al día de hoy, es sabido por las disciplinas proyectuales que los avances tecnológicos han permitido reducir ampliamente estas limitaciones. La incorporación de medios digitales CAD y 
fabricación digital CAM, transforman los modos de hacer en lo que Chiarella (2017) denomina pensamiento gráfico aumentado. Frente a estos escenarios, desde hace varias décadas, algunas voces vienen llamando la atención sobre los cambios que las nuevas tecnologías producen en los modos de hacer y pensar en diseño. Ya en 1994, Bernhard Bürdek advertía sobre los cambios metodológicos, teóricos y epistémicos producidos por la llegada de la microelectrónica y por ende, por la llegada de la inmaterialidad (p120). Böhme González y Barentin Calvo (2014) consideran que el desarrollo de competencias avanzadas en computación se ha vuelto un objetivo global en la formación de profesionales productivos y de calidad en esta nueva era. Siendo este enfoque consecuente con las necesidades formativas de diseñadores industriales que, como hemos visto, se ven enfrentados a cambios científicos-tecnológicos, sociales y económicos constantes. Como consecuencia de lo anterior, se identifica que el número de disciplinas que se interrelacionan dentro del campo de actuación del diseño cada vez es mayor, especialmente en los aspectos conceptuales y proyectuales. Sin embargo, a diferencia de lo que sucedía durante las experiencias académicas de la modernidad, los proyectos educativos ofrecidos por universidades nacionales argentinas, no se han actualizado frente a estas nuevas formas de conocer en diseño. Es necesario reconocer que actualmente existe una debilidad en la selección de conocimientos básicos para la formación de diseñadores industriales, como así también en los modos en que estos son organizados, articulados, profundizados.

\section{CULTURA MAKER. IDENTIDADES Y SIGNIFICACIONES}

A las experiencias Maker las caracteriza el uso de medios digitales, especialmente la informática y la manufactura digital para la resolución de problemas reales y contextuales a través de la fabricación de dispositivos, es decir, a través del hacer. Sobre estas experiencias existe una mirada extendida según la cual se las percibe como "innovadoras", "abiertas", "colaborativas", "flexibles, "integrales", "democráticas", en oposición a las instituciones universitarias percibidas como "rígidas", "disciplinadoras", "anacrónicas", "estratificadas", "conservadoras" ante los cambios (Chris Anderson, 2012). A raíz de ello, para los fines de éste artículo, los medios digitales son adoptados bajo una perspectiva cultural y procesual que evita reducirlos a meros instrumentos técnico-funcionales. Son considerados por sus efectos transformadores de las capacidades mentales humanas gracias a la ampliación de sus órganos sensoriales. (Maggio et al, 2014).

La cultura Maker es referida a menudo en relación a temas que van desde el regreso del hacer para el desarrollo de habilidades y destrezas, hasta la reconexión con nuestras raíces más humanas. ¿Qué relación tiene el desarrollo de dispositivos con nuestras raíces? En su libro, Chris Anderson (2012) nos explica que el ser humano, desde sus orígenes, tuvo la necesidad de hacer cosas, desde cocinar, hasta hacer su propia ropa. Desde niños jugamos a hacer cosas y, en el transcurso del tiempo, las invenciones y descubrimientos se han basado en el hacer.

Los inicios del movimiento Maker se remontan al movimiento cultural del "hágalo usted mismo" (en inglés
"Do It Yourself" - DIY) en USA, que se popularizó en los años cincuenta, en gran medida debido al retorno de los soldados luego de la segunda gran guerra. Ellos encontraban un pasatiempo que les permitía superar los recuerdos trágicos y las dificultades económicas y, los más jóvenes, muchas veces como símbolo de rebeldía, encontraban formas creativas de poder materializar sus expresiones artísticas. Así, el movimiento Maker se manifiesta en distintas actividades que van desde las artesanías hasta la música. Inclusive, desde aquellos años, ya existían comunidades en torno a publicaciones de carácter tecnológico para aficionados, como Popular Mechanics, Popular Electronics, entre otras.

Artesanos, diseñadores, artistas, reparadores, aficionados e inventores pueden ser considerados makers o hacedores. Como Chris Anderson (2012) dice: "Todos somos makers al nacer." En términos generales, un maker es alguien que extrae identidad y significado del acto de la creación. Lo que distingue a los makers contemporáneos de inventores y "DIYers" de otras épocas, son las potencialidades que obtiene mediante el uso de nuevas tecnologías y de una economía globalizada, tanto para conectarse y aprender, así también como medio de producción y distribución. Los softwares de diseño permiten dibujar, modelar, y simular los proyectos, reduciendo al mismo tiempo los plazos productivos como así también la curva de aprendizaje para utilizar herramientas de producción hogareñas. Los makers tienen acceso a materiales sofisticados y piezas de máquinas de todo el mundo. Foros, redes sociales, listas de correo electrónico y sitios de publicación de video permiten formar comunidades, hacer preguntas, colaborar, compartir resultados e iterar para alcanzar nuevos niveles de desempeño. Se podría pensar entonces que el movimiento Maker está basado en el 'hobby'. Sí, esencialmente son hobbies, pero éstos están pasando de ser una actividad de recreación, a una nueva fuerza económica que apunta a nichos de mercado bajo lo que se denomina "long tail" (Anderson, C; 2012).

Según algunos expertos, la cultura Maker podría representar una nueva revolución industrial, una nueva forma de fabricar en pequeñas empresas o grupos de individuos que se dedican a hacer sus propios productos. Más que una revolución se trata de una transformación, la posibilidad de inventar un "nuevo mundo" de artesanos, hackers y "manitas". (Hatch, 2014). Pero, independientemente de lo que signifique este movimiento en la economía global, lo que nos debe interesar es esta "cultura del hacer". Su ideología activa y participativa que lo impregna, su potencial para integrar formas y entornos de aprendizaje y su poder transformador para una educación ubicua. La energía y las herramientas de innovación y creatividad del movimiento Maker se presentan como instrumentos transformadores que están afectando las propuestas educativas a nivel global. Este hecho, que ciertos autores clasifican como la tercera revolución industrial, no sólo ha llamado la atención de aficionados, profesionales e industrias, sino que también incide en el campo educativo. La hipótesis que planteamos se refiere a que tal revolución es la manifestación emergente, en lo que se refiere a modos de enseñanza, de un cambio tecnológico. 


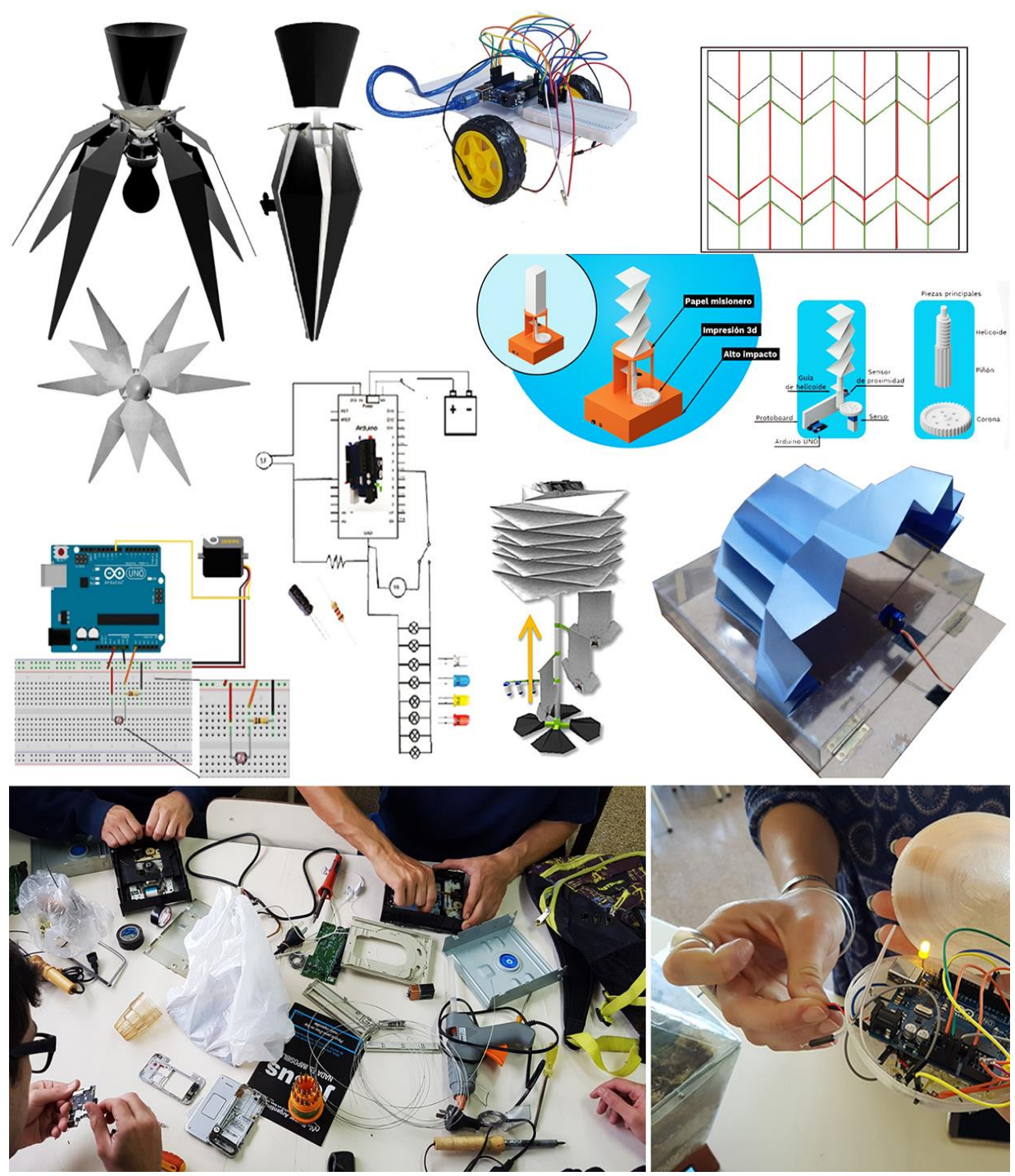

Figura 1: Composición síntesis que expone los procesos de exploración y algunos de los resultados alcanzados por los estudiantes.

\section{DISPOSITIVOS REACTIVOS}

A raíz de las nociones desarrolladas, hemos diseñado una práctica de enseñanza a modo de experimentación de algunos principios, ideas, teorías propuestas por el movimiento maker y el método de Aprendizaje Basado en Problemas (ABP). Se explora entonces la posibilidad de dar respuesta a los contenidos tecnológicos actualmente demandados y a la problemática curricular presente en torno a la integración y síntesis de conocimientos. Apoyados en el pensamiento interdisciplinar propio del diseño industrial, el trabajo colaborativo y el acercamiento a las demandas locales, se exploran respuestas más certeras frente a los requerimientos actuales y futuros de la profesión.
La experiencia de cátedra fue realizada dentro de la asignatura Introducción a los Medios Digitales (IMD) de la Licenciatura en Diseño Industrial de FADU UNL que cuenta con un cursado de $2 \mathrm{hs}$ semanales, se dicta en el cuarto cuatrimestre, formando parte del segundo nivel de la carrera, perteneciendo aún al ciclo básico de la misma. Cuenta con un promedio de 70 estudiantes que apenas se han iniciado en el uso de instrumentos relacionados a los medios digitales (software de diseño y equipamiento de fabricación digital) y no poseen conocimientos en torno a la microelectrónica y programación.

La práctica fue diseñada como ejercicio único en el cuatrimestre, subdividido en seis etapas. La consigna giró en torno al diseño y fabricación de lo que la cátedra define como "Dispositivos Reactivos". Los mismos se definen 
como artefactos que realizan un determinado tipo de comportamiento debido a un estímulo predefinido por el estudiante y fabricado por él (Chiarella et. Al, 2016). Para el desarrollo de los mismos se utilizaron conocimientos provenientes de distintas ciencias y oficios relacionados al uso de microcontroladores, sensores y actuadores, la fabricación digital y los Softwares CAD.

La propuesta de enseñanza fue diseñada considerando el atravesamiento de tres estrategias: el ABP, la enseñanza STEM (Ciencia, Tecnología y Matemáticas en inglés) y la técnica del "rompecabezas". El ABP nos orienta hacia un enfoque de enseñanza centrado en el estudiante (LCT). La enseñanza STEM nos brinda los recursos más formales que posibilitarán la implementación de una 'enseñanza Maker' en un ejercicio dentro del ámbito de la educación formal superior. (Felder \& Brent, 2016). Finalmente, en un nivel más operativo, se utilizó la técnica del "Rompecabezas" (Jigsaw) ideada en los setenta por el profesor Elliot Aronson (Gutiérrez N, 2014). El Rompecabezas es una técnica de aprendizaje cooperativo basada en la investigación. La secuencia de pasos da estructura a la actividad y genera una interdependencia entre los estudiantes. Este ejercicio se apoya entonces en estas metodologías articuladas a la enseñanza STEM y la potencialidad de la cultura Maker, para materializar los prototipos de cada proyecto y desarrollar así nuevas habilidades en torno a su formación.

Para el devenir del ejercicio se desplegaron los siguientes pasos modalizando la técnica del Rompecabezas:

1- El curso se dividió en grupos de 5 a 6 estudiantes.

2- Cada grupo propuso un líder que surgió de su dinámica interna.

3- Los contenidos pertinentes a la asignatura se dividieron en los tres sistemas a desarrollar: microcontroladores, sistemas CAD y fabricación digital.

4- Cada grupo dividió sus integrantes según los tres sistemas que deberán aprender.

5- Se configuraron "grupos de expertos" (dos miembros) que discutieron y ensayaron las exposiciones que posteriormente realizaron en sus grupos originales.

6- Los integrantes de los grupos de expertos retornaron a sus grupos originales y expusieron lo aprendido de cada uno de los temas. De éste debate surgió la temática de diseño a abordar en cada grupo considerando problemáticas sociales.

7- Los docentes tuvieron la tarea de moderar los pasos para así poder lograr ésta dinámica de trabajo

8- Evaluación. Cuando finalizó el trabajo cada grupo debió exponer sus desarrollos al resto. Se utilizó el formato PechaKucha (20 slides en 20 seg.) más un minuto para el espacio de preguntas donde se verificó que se hayan cumplido los objetivos de la ejercitación.

\section{REFLEXIONES SOBRE LA EXPERIENCIA}

Durante todo el proceso se realizaron tres encuestas como modo de registro y recolección de datos. La primera fue realizada posterior al lanzamiento de la actividad y buscó conocer la recepción de la propuesta por parte del alumnado. La segunda fue luego del desarrollo de los contenidos para indagar en torno a las habilidades y destrezas desarrolladas. La última fue al final y perseguía conocer el grado de satisfacción de la propuesta. Durante la primera encuesta pudimos conocer el grado de motivación despertado como así también ciertas resistencias. Respecto a la interrogante ¿Cómo te sientes en cuanto a la propuesta? Los estudiantes se mostraron mayoritariamente receptivos:

- Es una propuesta interesante, ya que por comentarios de estudiantes de años anteriores, la metodología se volvió más interesante porque nos pone en contacto con herramientas que vamos a terminar usando a lo largo de la carrera.

También encontramos ciertas resistencias e inquietudes:

- Creo que me va a resultar difícil llevar a cabo el trabajo práctico porque nunca hice actividades de ese tipo, pero espero poder resolverlo.

Luego del lanzamiento del ejercicio se realizaron las primeras etapas, en donde los grupos de seis tuvieron que dividirse en tres para poder formarse por separado en los distintos temas. De esta manera generamos la interdependencia entre los miembros, potenciando así la participación de todos. Las primeras clases fueron de contenidos distintos por lo que cada grupo docente dictaba en simultáneo y haciendo uso de tres proyectores las clases requeridas. Luego de haber formado a los subgrupos de expertos, la actividad continuó con el rearmado de los grupos originales de seis miembros, cada cual ya formado en temas específicos. Dentro de esta etapa se realizó la segunda encuesta en torno a las habilidades y destrezas obtenidas. Frente a la pregunta ¿Cuál es su opinión respecto a la estrategia de dividir los grupos para trabajar por separado temas distintos? Se obtuvo una valoración positiva, aunque en la práctica se identificaron dificultades:

- Está bueno. Permite ahondar más en cada disciplina, y genera otra dinámica a la hora de juntarse a trabajar con el grupo completo. Ayuda mucho que cada disciplina se componga por pares.

- Venimos bien. Es difícil porque es un grupo numeroso, y cada uno cursa distintas materias. Desgraciadamente las dos horas curriculares no dejan mucho espacio para avanzar en clases.

A pesar de ello, frente a la pregunta: ¿De cuántos integrantes cree que deberían ser los grupos? La mayoría sigue prefiriendo grupos de seis miembros.

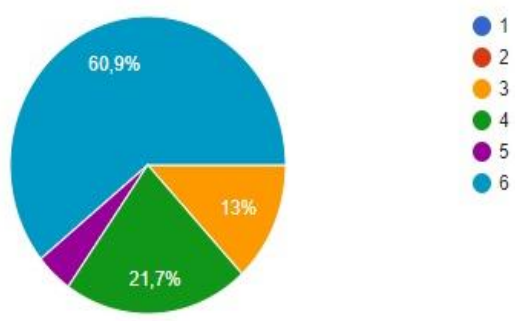

Finalmente frente a la pregunta: ¿Cuál es el grado actual de conformidad con la propuesta? La recepción es alta y satisfactoria. 


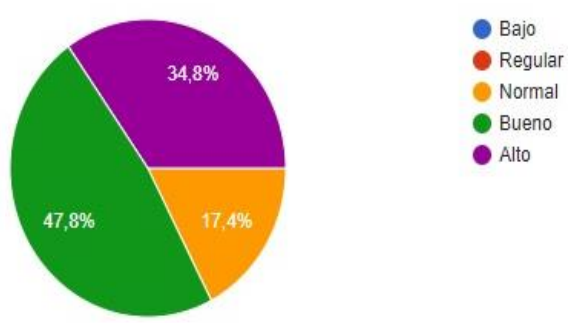

La última etapa del trabajo consistió en el diseño y fabricación de los dispositivos. Aquí surgieron situaciones de índoles distintas que los docentes tuvieron que sobrellevar con adaptabilidad y resiliencia. Hacia el final las opiniones fueron más heterogéneas que al principio. Cada grupo de estudiantes y cada estudiante en sí vivió la experiencia de forma particular, quedando registradas en la última encuesta. Frente a la pregunta ¿Cómo le resultó el trabajo en grupo? Surgieron respuestas tanto a favor como de resistencia:

- Muy bueno, aunque al ser tantos en el grupo siempre nos terminamos dividiendo el trabajo en vez de juntarnos todos a trabajar.

Sobre la pregunta ¿Qué opinión tiene sobre que el trabajo práctico haya sido basado en los principios de la cultura Maker? Se concluye que ha sido un acierto gracias a la recepción obtenida:

- Me parece una idea genial, con mucho contenido y a su vez nos ofrece un mundo de posibilidades sobre nuestro futuro. En mi opinión, una materia de nuestra carrera tenía que tener un tema abordado de la manera que se hizo.

También se indagó sobre la utilidad de los contenidos y prácticas elegidas en torno a las preguntas ¿Cree que los contenidos aportan a la formación del diseñador? ¿Los cree pertinentes para el futuro trabajo profesional? Aquí también la recepción fue mayormente positiva:

- Definitivamente, sí. Creo que fue uno de los trabajos más completo que realicé, si no el más el completo, a lo largo de la carrera.

- Claro que sí, los diseñadores deberían estar cada vez más conectados con el mundo digital y sobretodo con las nuevas técnicas de trabajo (modelado, programar, impresión 3D, etc).

Finalmente realizamos una pregunta abierta, a modo de cierre, para que puedan expresar su grado de conformidad con el proceso general, a través de la consigna: "Opine sobre la propuesta en general. Sería interesante que incluyan comentarios sobre el trabajo de los docentes. A fin de seguir construyendo sobre la experiencia. Gracias". Esta consigna disparó toda clase de respuestas, muy valiosas para reflexionar sobre las innovaciones perseguidas. En general las respuestas son positivas en su mayoría, por un tema de extensión se citan solo algunas de ellas. Todos concordamos que el tiempo es escaso para una práctica compleja, pero confiando en el proceso se logra el cumplimiento de los objetivos. Queda registro de los estudiantes de cómo han vivido el acompañamiento de los docentes, la resolución del dispositivo abordado y las dinámicas de grupo:

- Me hubiera gustado en ocasiones un poco más de orden en el aula, ya que muchos hacían lo que querían, con respecto a los profesores no tengo ninguna queja ya que siempre estuvieron cuando lo necesitamos incluso fuera del horario de la cátedra, lo que sí me parece que hay que mejorar es la ayuda al estudiante al momento de hacer los prototipos, por ejemplo con la impresora 3d que no haya estado en funcionamiento, por lo menos ninguno de mi grupo alguna vez trabajo con la impresora de la facultad y parece que solo unos pocos tienen el privilegio de hacerlo.

- El trabajo fue súper interesante, respecto también a los trabajos de los años anteriores. Me parece bueno para proporcionar una base y dar una idea a los alumnos de las cosas que se pueden llegar a hacer, quizás a alguno le interesa y siga investigando por su cuenta.

En síntesis, se pudo valorar como positiva la experiencia realizada. El grado de conformidad sobre las personas encuestadas fue mayoritario, aunque no todo el alumnado participó de las mismas (alrededor de un $50 \%$, es decir, unos 40 estudiantes). A pesar de ello, fueron aisladas las opiniones negativas, existiendo claro variedad de aportes para su mejoría. Para nuestra sorpresa, los estudiantes se mostraron plenamente expectantes frente a la propuesta y se mantuvieron motivados durante toda la práctica. Esto nos da indicios de los necesarios ajustes que las prácticas curriculares requieren.

Los medios digitales y las nuevas formas de recibir, procesar, crear y distribuir información con su uso han generado nuevos entornos de aprendizaje, modificado sustancialmente los existentes. La experiencia aquí presentada propone, al igual que los casos relevados, una práctica transformadora a modo de arietes que venzan las barreras curriculares. De momento parece claro que las competencias digitales básicas se desarrollan mejor fuera que dentro de las universidades nacionales, pero en los entornos de aprendizaje tecnológico informal no suele profundizarse sobre la importancia de la tecnología digital en nuestras sociedades ni todas las prácticas favorecen la educación integral de los jóvenes.

\section{PROPONIENDO EL CAMBIO}

El mundo se ha expandido, enriquecido y complejizado por una abundancia de tecnologías digitales. Lo que implica que gran parte está configurado y controlado por programas, medios y comunicaciones digitales. En este contexto, es altamente probable que aquellos ciudadanos que no posean una serie de conocimientos, actitudes y saberes prácticos, constituyan una nueva clase de "analfabetos" de época. Las competencias necesarias hoy día en distintas áreas superan con creces la lectoescritura y el cálculo, lo que ha dado pie a que se hable de numerosas "alfabetizaciones". Tyner, Gutiérrez y Alba (2015) proponen el término "multialfabetizaciones", para referirse a los aprendizajes y competencias necesarios para la comunicación multimodal propia de una sociedad tecnológica, global y diversa en lo cultural y lo social. Los cambios acelerados y la incertidumbre que definen el contexto actual demandan permanentes reflexiones, innovaciones y/o relecturas de la realidad, el campo educativo no queda exento. Frente a estos desafíos, las 
tecnologías nacidas en el mismo núcleo del movimiento Maker como microcontroladores e impresoras 3D de bajo costo, permiten disminuir lo que Mitchel Resnick (2001) denomina como "brecha tecnológica". La clave educativa está en relación con ayudar a formar personas que puedan interactuar con la sociedad actual, en función de darles libertad, para que puedan desarrollarse de una mejor manera y a la vez insertarse apropiadamente, en el mundo laboral y social. García, A; et Al (2012) reconocen la importancia de las relaciones sociales y la interacción con el otro en la adquisición de conocimiento; saber trabajar en grupo para conseguir objetivos comunes aparece como una competencia transversal de aprendizaje en todos los niveles de enseñanza. En otras palabras, desde la relación con las tecnologías digitales, el desafío educativo se centra en formar personas proactivas, que interactúen con el mundo digital, no sólo consumiendo sus productos u objetos, sino también, que puedan crearlos y compartirlos.

Si nos limitamos a proponer prácticas que solo desarrollen proyectos a nivel gráfico, a pesar que las presentaciones incorporen modelos físicos (maquetas), estamos privando a nuestros estudiantes de los saberes que se desarrollan durante el hacer. A raíz de ello, consideramos necesario promover proyectos educativos para la formación de diseñadores que desarrollen el proceso completo sobre el diseño de un producto. Con esto queremos destacar la necesidad de realizar u aproximar la fabricación de los mismos. Cómo hemos visto, pensar y hacer en diseño no se encuentran escindidos. Si dichas prácticas, además, incorporan el trabajo colaborativo como modo de construir socialmente los conocimientos, estas potenciaran el desarrollo de nuevos saberes. EI ABP como plataforma permite conservar el núcleo fundamental de la formación de diseñadores que es la del "taller de diseño", en donde se integran los conocimientos disciplinares en la resolución de un problema propuesto. De esta forma, enfocando las acciones, es posible potenciar la transposición didáctica (Chevellard, 2005), permitiendo la articulación de los conocimientos necesarios requeridos para resolver un problema complejo.

Estas estrategias hacen posible el logro de propuestas de enseñanza innovadoras, como así también la adquisición de los conocimientos actualmente requeridos. Esperamos que las reflexiones presentadas contribuyan al pensamiento de prácticas innovadoras que apunten a vencer las barreras curriculares como un diseño para la transformación.

\section{REFERENCIAS}

Anderson, C (2013). Makers: The New Industrial Revolution. Crown Business.

Böhme, LFG; Barentin CC (2014). Desarrollo de Competencias Avanzadas en Computación en la Formación de los Arquitectos Latinoamericanos del Siglo XXI. En SiGraDi. São Paulo: Blucher.

Bürdek, B (1994). Diseño: historia, teoría y práctica del diseño industrial. Ed Gustavo Gili. Barcelona

Burton, C. (1982). El sistema de educación superior. Una visión comparativa de la organización académica. UNAM, México.

Canciano, E. (2013). Lo escolar fuera de la escuela Un estudio acerca de los modos de organización de la acción educativa destinada a niñas, niños y adolescentes en ámbitos situados fuera del sistema educativo. FLACSO, Argentina.

Camilloni, A. W. (2016). Tendencias y formatos en el currículo universitario. Instituto de Investigaciones en Ciencias de la Educación. Facultad de Filosofía y Letras, UBA.

Chiarella, M; et. Al. (2016). Cultura Maker. Dispositivos, Prótesis Robóticas y Programación Visual en Arquitectura y Diseño para eficiencia energética. SIGraDi. São Paulo: Blucher.

Chiarella M. (2017). Pensamiento gráfico aumentado. Revista de la FADU N 15 p40-47. Montevideo, Uruguay.

Coscarelli, MR; Picco, S. (2009). Protocurriculum: sentidos dispersos en un campo complejo. En La extensión universitaria: sujetos, formación y saberes. UNLP. p. 63-85.

De Alba, A. (1991). Currículum: crisis, mito y perspectivas. Ed. Miño y Dávila. Buenos Aires, Argentina.

De Alba, Alicia (2007): Curriculum-Sociedad. El Peso de la Incertidumbre, la Fuerza de la Imaginación. Itinerarios Educativos, 1(3), 117-118.

De Souza silva, J (1999). ¿Una época de cambios o un cambio de época? Revista Recursos Naturales y Ambiente $\mathrm{N}^{\circ} 28$.

Díaz Barriga, A. (2007). Didáctica y curriculum. Ed Paidos Ibérica.

Donoso, CSF (2016). El Diseño Industrial; las fronteras confusas de la creatividad. UNer, Argentina

Edelstein, G (2000). El análisis didáctico de las prácticas de la enseñanza. Una referencia disciplinar para la reflexión crítica, en la Revista del IICE Nro. 17, Bs. As., Miño y Dávila.

Felder, R.M., \& Brent, R. (2016) Teaching and learning STEM: A practical guide, Jossey-Bass.

Furlan, A (1992). Curriculum y condiciones institucionales. Cuadernos pedagógicos universitarios. UCOL, México.

García Canclini, et. Al. (2012) jóvenes, culturas urbanas y redes digitales. Madrid: Fundación Telefónica.

García, A; et Al (2012) La metodología a metodología del aprendizaje colaborativo a través de las TIC: una aproximación a las opiniones de profesores y alumnos. Revista Complutense de Educación Vol. 23 Núm. 1 p161-188

Gutiérrez N, C. (2014) análisis comparativo de metodologías de aprendizaje colaborativo, jigsaw y aprendizaje basado en problemas, haciendo uso de objetos de aprendizaje reutilizables, para el aprendizaje de la geometría, en alumnos de primero medio. FCS, Universidad de Chile.

Hatch, M. (2014). The Maker Movement Manifiesto. McGraw-Hill Education. ISBN: 978-0-07-182113-1.

Hidalgo, C. (2013). Cómo salir del encierro en disciplinas: competencias, interdisciplina, redes de conocimiento. En Tensiones entre disciplinas y competencias en el currículum universitario. Ediciones UNL.

Maggio, M. (2014). Enriquecer la enseñanza superior: búsquedas, construcciones y proyecciones En: InterCambios, $n^{01}$, junio.

Resnick, M. (2001). Closing the Fluency Gap. En Communications of the ACM Vol. 44, No. 3, p144.

Tesconi, S. (2015). Crear artefactos para generar conocimiento compartido. Comunicación y pedagogía: Nuevas tecnologías y recursos didácticos, (283), p40-47.

Tonelli, I. (2009). Modelo Epistemológico de las relaciones entre el pensar y hacer en el proceso de enseñanza aprendizaje de la Arquitectura. Revista Nova scientia, Vol. 2, №. 3.

Tyner, K; Gutiérrez MA; Torrego González, A. (2015). "Multialfabetización" sin muros en la era de la convergencia. La competencia digital y "la cultura del hacer"como revulsivos para una educación continua. Revista de Currículum y Formación de Profesorado, vol. 19, núm. 2, pp. 41-56. 